\title{
Triggers, modes of presentation and outcomes of stress cardiomyopathy
}

\author{
Ranasinghe RBD ${ }^{1}$, Abeysiriwardana $\mathrm{DA}^{1}$, Sathananthan $\mathrm{PP}^{1}$, Ranasinghe RATK ${ }^{2}$, \\ Muthukumarana $\mathrm{PAI}^{1}$
}

Journal of the Ceylon College of Physicians, 2020, 51, 139-143

\begin{abstract}
Stress cardiomyopathy is an underdiagnosed condition characterized by transient hypokinesia/ akinesia of left ventricle following a physical or psychological trigger in the absence of occlusive coronary artery disease.
\end{abstract}

Seven patients managed as definitive or possible stress cardiomyopathy at the Teaching Hospital Karapitiya, Sri Lanka over a period of one year are included in this case series. Five patients underwent coronary angiography with or without ventriculography leading to a definite diagnosis. The other two patients were diagnosed based on their echocardiography findings alone. All seven patients were women and the onset was triggered by various physical and emotional triggers including infections, per cutaneous intervention, adrenaline injection, surgery, heated argument and sudden death of a loved one.

Stress cardiomyopathy has a significant variation of triggers, modes of presentation and outcomes. We discuss seven cases of this condition to help clinicians to better understand and manage this treatable condition.

Key words: stress cardiomyopathy, stress induced cardiomyopathy, Takotsubo cardiomyopathy, reversed Takotsubo cardiomyopathy, transient hypokinesia

\section{Introduction}

Stress cardiomyopathy, also known as Takotsubo cardiomyopathy or broken heart syndrome is a pheno- menon characterized by transient hypokinesia, akinesia or dyskinesia of left ventricular segments which may extend beyond specific coronary artery territory distribution. Evidence from animal studies suggest that this phenomenon is a result of intense sympathetic activation, coronary vasospasm or direct catecholamine toxicity. ${ }^{1-3}$ Stress cardiomyopathy primarily affects postmenopausal woman who present in a manner identical to that of acute coronary syndrome (ACS) usually following a psychological and sometimes a physical stress. It's usually a transient condition which completely recovers within 4-6 weeks with supportive and conservative treatment. Recurrences are known to occur in about $10 \%$ of patients. ${ }^{2}$

ECG abnormalities usually resembles that of ST elevation myocardial infarction and are associated with elevated troponin and BNP levels. However, there is no evidence of obstructive coronary artery disease in angiography. ${ }^{1}$ Co-morbid conditions such as obesity, diabetes, hypertension, smoking, dyslipidaemia and alcohol abuse are associated with both ACS and stress cardiomyopathy. ${ }^{1}$ Psychiatric, neurological disorders and malignancies are also found to be associated with stress cardiomyopathy.

There are several subtypes of this condition categorized according to wall motion abnormalities seen in echocardiography or ventriculography. ${ }^{1}$

1. Apical type - hypokinesia of mid and apical ventricular segments often associated with hyperkinesia of basal segments. This is the commonest type.

${ }^{1}$ Cardiology Unit, Teaching Hospital, Karapitiya, Sri Lanka, ${ }^{2}$ Colombo South Teaching Hospital, Kalubowila, Sri Lanka.

Correspondence:RBDR, e-mail: bhathiyarbd@yahoo.com

(iD) https://orcid.org/0000-0002-7546-346X

Received 07 May 2020, accepted 01 August 2020.

This is an open-access article distributed under the terms of the Creative Commons Attribution License, which permits unrestricted use, distribution, and reproduction in any medium, provided the original author and source are credited. 
2. Midventricular type - hypokinesia of mid ventricular segments with sparing of apex and base of left ventricle

3. Basal type - hypokinesia of basal segments with sparing of mid and apical segments

4. Focal type - hypokinesia of isolated left ventricular wall segment (commonly anterolateral segment)

5. Global type - global hypokinesia

\section{Case descriptions}

\section{Case 1}

A 71-year-old previously well woman presented with left sided constricting type of chest pain for one day duration following a heated argument. Pain was associated with effort dyspnoea and orthopnea. Her ECG showed inverted $T$ waves in anterior leads compatible with LAD territory ischemia. Echocardiography revealed hypokinesia in the mid wall and apical segments with preserved systolic function in basal segments compatible with apical type stress cardiomyopathy. Her EF was 35\%. Troponin was positive and she was initially managed as NSTEMI. Subsequent coronary angiogram and left ventriculogram showed normal coronary arteries. She was treated with optimal heart failure therapy. Follow up echocardiography done six weeks later revealed complete recovery of left ventricular function.

\section{Case 2}

A 25-year-old previously healthy woman, mother of a six-month-old baby was admitted following anaphylaxis to wheat flour. She developed constricting type chest pain after intramuscular adrenaline injection. Her ECG showed T inversions in V3 to V6 leads and troponin I was negative. Echocardiography done at the local hospital revealed moderate left ventricular systolic dysfunction, EF of $40 \%$ with hypokinetic segments and managed as unstable angina. She was transferred to our hospital for coronary angiogram. Her detailed echocardiography showed hypokinesia in basal wall with preserved contraction in mid and apical segments of left ventricle compatible with reversed type stress cardiomyopathy She underwent coronary angiogram and left ventriculography which revealed normal coronary arteries. Her left ventriculogram confirmed the echocardiographic findings. She made a complete recovery with conservative treatment. Follow up echocardiography done six weeks later showed complete recovery of left ventricular function.

\section{Case 3}

A 35-year-old mentally subnormal woman with history of epilepsy (off treatment for last 5 years), was admitted to local hospital with status epilepticus. She was intubated and transferred to our hospital for further management. The ECG showed ST segment depressions in inferior leads and troponin I was positive. Echocardiography showed mid ventricular hypokinesia with preserved basal and apical function compatible with mid ventricular type stress cardiomyopathy. Her EF was $35 \%$. The coronary angiogram was normal and her ventriculogram confirmed the diagnosis. She had complete recovery with supportive treatment. Follow up echocardiography done two weeks later showed a normal study.

\section{Case 4}

A 28-year-old woman with posterior fossa tumor (pilocytic astrocytoma) was admitted with headache followed by altered level of consciousness. Her noncontrast CT brain revealed ventriculomegaly which was suggestive of acute hydrocephalus. She was transferred to TH Karapitiya for neurosurgical management, where she underwent external ventricular drainage following which her conscious level improved. On postoperative day 1 in the ICU she developed hypotension with tachycardia. Her electrocardiogram showed diffuse $T$ wave inversions. Chest $X$-ray revealed white-out right lung. She was started on inotropes and treated as for possible aspiration pneumonia. Her 2 D echocardiogram revealed hypokinetic apex and mid wall with preserved base compatible with typical stress cardiomyopathy. She had severe left ventricular dysfunction with an EF of $30 \%$ and hypotension was corrected with ionotropes. She was given IV frusemide infusion and repeat chest radiography 24 hours later showed rapid resolution of pulmonary shadows suggesting unilateral pulmonary oedema. She was treated conservatively with optimal heart failure therapy. Subsequent echocardiography done revealed total recovery of LV function. She subsequently underwent successful excision of the cerebellar tumor and made a complete recovery.

\section{Case 5}

A 65-year-old woman with hypertension, dyslipidaemia and stable angina with single vessel disease underwent elective PCI to LAD with a drug eluting stent. The procedure was uneventful. She was transferred for coronary care unit for post-operative observation. Six hours later, she developed sudden breathlessness, chest pain and hypotension. ECG showed $\mathrm{T}$ wave changes in anterior leads. $2 \mathrm{D}$ echocardiogram revealed preserved basal function with hypo kinetic mid and apical segments with an EF of $35 \%$. She underwent repeat coronary angiogram with the 
suspicion of stent thrombosis. The angiogram showed patent stent with normal coronary flow and her ventriculogram confirmed typical form of stress cardiomyopathy (Figure 1). She was managed conservatively with heart failure medications. Her follow up echocardiogram in 6 weeks later showed complete recovery of left ventricular function.

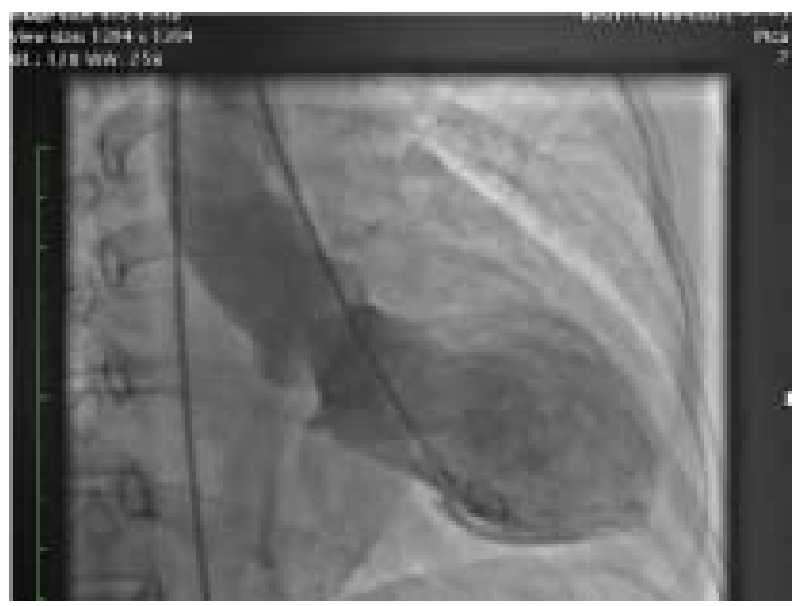

Figure 1. Ventriculography of Case 5.

\section{Case 6}

A 36-year-old mother of two children was admitted with fever, headache, arthralgia and myalgia for 3 days with positive dengue NS1 antigen report. Her platelet count was $130,000 / \mu \mathrm{L}$ and the abdominal ultrasound scan did not show evidence of fluid leakage. On the same day morning she got to know about the sudden death of her father. She subsequently developed a cardiac arrest which was resuscitated for 15 minutes and was intubated and transferred to ICU for further care. She was treated for cardiogenic shock with 4 inotropes including noradrenaline, dobutamine and dopamine. Her echocardiogram showed characteristic features of apical cardiomyopathy with hypokinetic apical and mid wall with preserved basal function. Unfortunately, she succumbed to multiorgan failure despite optimal medical management. Probable cause of death was given as apical type stress cardiomyopathy.

\section{Case 7}

A 59-year-old woman with dyslipidaemia who had fever for 2 days developed chest pain suggestive of ACS. Her ECG done by a general practitioner had revealed right bundle branch block. Later she had a cardiac arrest out of hospital which was resuscitated upon arrival to ETU. A 2 D echocardiogram revealed mid and apical wall hypokinesia with preserved LV basal function. (EF-35\%). A coronary angiogram was done which showed minor coronary artery disease with normal coronary flow. Her mycoplasma antibody test was positive. It's very likely that this infection could have been the trigger of stress cardiomyopathy. She was hypotensive and was treated with inotropes and heart failure medications. However, she subsequently died of multi organ dysfunction.

\section{Discussion}

Stress cardiomyopathy is a phenomenon characterized by left ventricular dysfunction in the absence of occlusive coronary artery disease typically following an emotional or physical trigger. Symptoms such as chest pain and breathlessness can mimic an acute coronary syndrome. Electrocardiographic abnormalities are common in patients with stress cardiomyopathy. ST segment elevation is the commonest ECG abnormality typically involving anterior leads whereas ST depression is less common. Other findings include QT prolongation, T wave inversion, abnormal $Q$ waves and nonspecific changes. Our patients mostly had $\mathrm{T}$ wave inversions.

Slight elevations of cardiac troponin are seen in most patients with stress cardiomyopathy. ${ }^{4}$ However, degree of troponin elevation is not proportional to the degree of symptoms and ECG changes. This disproportionate troponin elevation should lead to suspicion of stress cardiomyopathy. Brain natriuretic peptide levels are elevated in some patients. However, this test was not done in our patients.

Echocardiography with or without ventriculography reveals the features of stress cardiomyopathy which is characterized by mid and apical segment hypokinesia of left ventricle with preserved or hypercontractile basal segments. This type of regional wall motion abnormalities extends beyond the supply territory of any single coronary artery. Ventriculogram done at the time of coronary angiogram will confirm the echocardiographic findings. However, for a definitive diagnosis it is essential to exclude obstructive coronary artery disease. ${ }^{1,3}$

Although stress cardiomyopathy is reported to be common in post-menopausal women 4,4 out 7 patients in our case series were young women. All seven patients were found to have an identifiable precipitant as mentioned in the Table 1. Four patients had apical/typical form of the cardiomyopathy while 2 patients had mid ventricular wall hypokinesia. One 


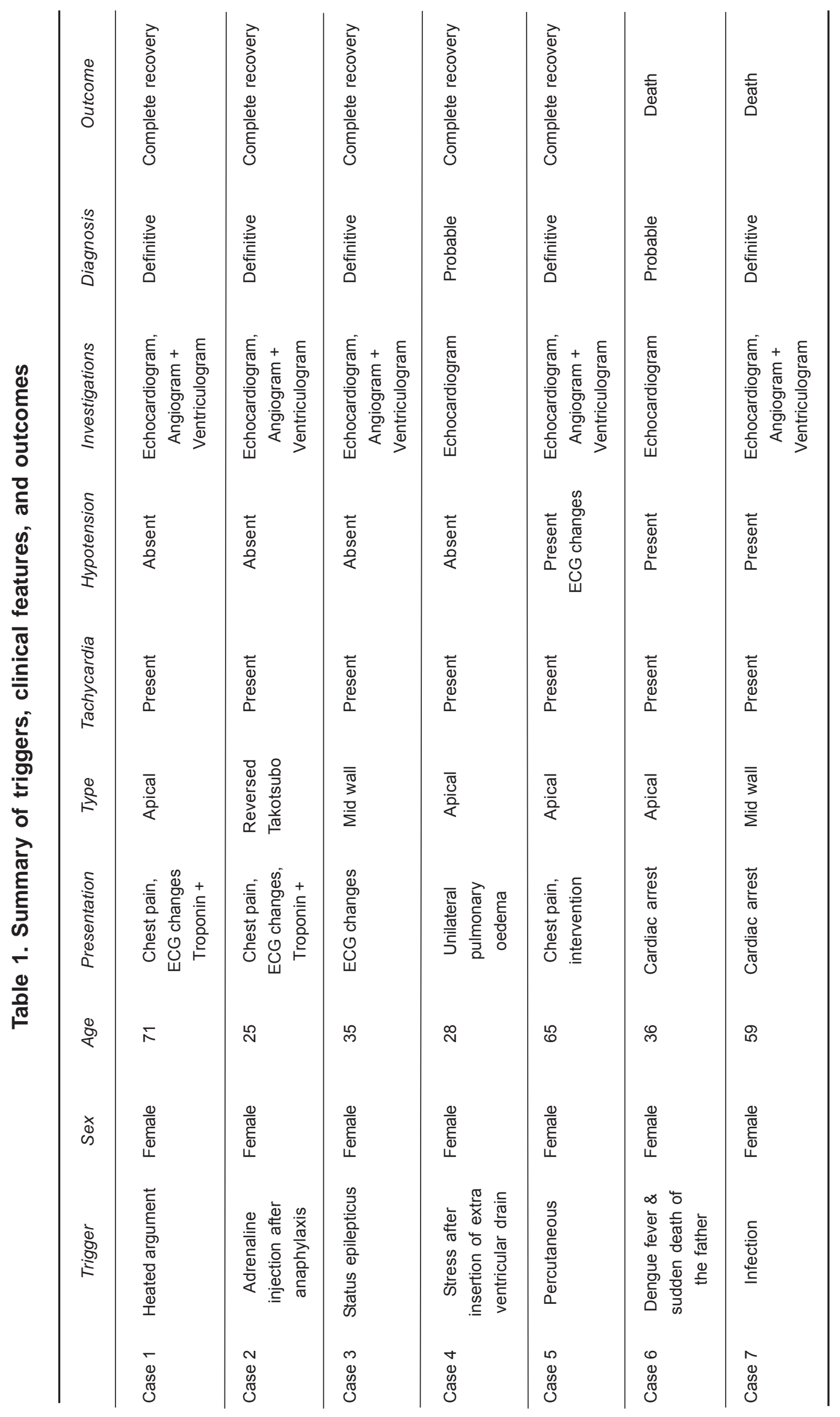


patient was diagnosed as Reversed Takotsubo Cardiomyopathy (basal hypokinesia). Four patients had hypotension; 2 of them died due to multiorgan dysfunction while the other 2 patients made a complete recovery. They were managed with inotropes to maintain blood pressure with close surveillance of fluid balance. Optimal heart failure medications were added including diuretics (frusemide and spironolactone), ACE inhibitors and beta blockers. Medications were stopped upon complete recovery of left ventricular function.

Two patients of the case series didn't undergo coronary angiography and were managed as probable stress cardiomyopathy. They were included in the case series solely based on the echocardiographic findings. Most of them were critically ill to mobilize.

This case series portrays different types of triggers and variety of presentations of stress cardiomyopathy. It can be concluded that this is an important condition which clinicians needs to conmsider in the differential diagnosis of ischaemic cardiac pain.

\section{Conflicts of interest}

There are no conflicts of interest for any of the authors.

\section{References}

1. Medina de Chazal H, Del Buono MG, Keyser-Marcus L, et al. Stress Cardiomyopathy Diagnosis and Treatment: JACC State-of-the-Art Review. J Am Coll Cardiol. 2018; 72(16): 1955-71. doi:10.1016/j.jacc.2018.07.072

2. Sharkey SW, Lesser JR, Zenovich AG, et al. Acute and reversible cardiomyopathy provoked by stress in women from the United States. Circulation 2005; 1; 111(4): 472-9. doi: 10.1161/01.CIR.0000153801.51470.EB. PMID: 15687136

3. Kato K, LyonAR, Ghadri JR, Templin C. Takotsubo syndrome: aetiology, presentation and treatment. Heart. 2017; 103(18): 1461-9. doi: 10.1136/heartjnl-2016-309783. PMID: 28839096.

4. Bybee KA, Kara T, Prasad A, Lerman A, Barsness GW, Wright RS, Rihal CS. Systematic review: transient left ventricular apical ballooning: a syndrome that mimics STsegment elevation myocardial infarction. Ann Intern Med. 2004; 141(11): 858-65. doi:10.7326/0003-4819-141-11200412070-00010. PMID: 15583228. 